\title{
Foodscapes, Finance and Faith: A Qualitative Investigation of Multi-Sectoral Stakeholder Perspectives on a New Mall and Supermarket in Kenya
}

\section{Pamela Wadende ( $\sim$ pamela.wadende@gmail.com )}

Kisii University College Faculty of Education and Human Resource https://orcid.org/0000-0003-08463977

\section{Oliver Francis}

University of Cambridge

\section{Rosemary Musuva}

Kenya Medical Research Institute

\section{Ebele Mogo}

University of Cambridge

\section{Eleanor Turner-Moss}

University of Cambridge

\section{Vincent Were}

Kenya Medical Research Institute

\section{Charles Obonyo}

Kenya Medical Research Institute

\section{Louise Foley}

University of Cambridge

\section{Research}

Keywords: Foodscape, Multi-sectoral Stakeholders, perspectives, supermarkets, Western Kenya, Socioecological model

Posted Date: June 1st, 2021

DOl: https://doi.org/10.21203/rs.3.rs-547640/v1

License: (c) (i) This work is licensed under a Creative Commons Attribution 4.0 International License. Read Full License 


\section{Abstract}

Introduction

The nutritional transition is fuelling a concerning rise in Non-Communicable Diseases (NCDs) in Low and Middle-Income Countries (LMICs). These countries lack strong health infrastructure capable of supporting the long-term and expensive medical treatment for those living with NCDs. It is important to identify stakeholders involved in influencing food retail and dietary choices as part of a population-level strategy to reduce the burden of NCDs in LMICs. The aim of this study is to explore stakeholder perspectives on the impacts of new and existing food retail on local diets in Kenya.

Methods

Regulatory and local community stakeholders from Kisumu and Homabay Counties of Western Kenya in this study responded to a semi-structured, open-ended interview schedule. We sought their perspectives on the impact of a proposed new mall and supermarket in Kisumu and existing supermarkets in Homabay on local dietary practices. Digital voice recorders captured the responses, which were transcribed verbatim then translated from Kiswahili or Dholuo languages into English. NVivo12 PRO software was used to analyse the coded responses using a thematic discourse analysis approach.

\section{Findings}

Both regulatory and local community stakeholders were similarly concerned that supermarkets make unhealthy food items (including fast food) more accessible and therefore easily incorporated into local diets, with the result that more people develop diet-related NCDs. At the same time, both groups of stakeholders indicated the usefulness of supermarkets as a reliable food source, albeit requiring that the shopper discern healthy from unhealthy food items. Overall, they were unsure whether such aspects as the pricing and convenience of supermarkets would be sufficient to cause the local population to stop patronising their usual small-scale food vendors, and had similarly mixed views on wider impacts on the local economy and livelihoods.

Conclusion

The prominent themes identified in our analysis were fear of fast food and health implications, supermarkets as reliable (but selective) food outlets, and uncertainty and differences of opinion about the potential mixed impacts of supermarkets on local communities. We found evidence of a multi-sectoral approach with a widely held interest in potential impacts on health.

\section{Background}

The growing burden and cost of managing NCDs on families, communities and the healthcare delivery system is substantial and has prompted a call for action at global, regional and national levels. ${ }^{1,2,3,4,5}$ Indeed, Dalal et al. ${ }^{6}$ note that in sub-Saharan Africa, the prevalence of hypertension stands at between 
$6 \%$ and $48 \%$, diabetes at $0 \%$ and $16 \%$, and obesity at $0.4 \%$ and $43 \%$. Data from demographic and health surveillance systems show increasing trends of NCD morbidity and mortality in sub-Saharan Africa., 3,4 5 Hospital-based studies also show increasing trends in adult admissions and deaths attributable to NCDrelated conditions. ${ }^{7,8}$

A few studies have particularly focused on unearthing the burden of NCDs in local Kenyan communities. Such studies include the Kenya Demographic and Health Survey (KDHS) ${ }^{9}$ of 2014, which noted that at least $9 \%$ of women and $3 \%$ of men have been told by a health care provider that they had hypertension and the percentages generally increased with age, wealth and education. A population-based household survey of 5000 participants in Nakuru town, Western Kenya, conducted by Mathenge and Kuper ${ }^{10}$, found $50 \%$ prevalence of hypertension, $7 \%$ diabetes, $13 \%$ obesity, and $21 \%$ of high cholesterol. Additionally, informal settlements and slums in Kenya's capital city, Nairobi, were the common site of surveys of NCD. In 2010, a population-based survey in Nairobi's Kibera slums found a $23 \%$ prevalence of hypertension and a $5 \%$ prevalence of diabetes. ${ }^{11,12}$ In the informal settlement/slums, Korogocho and Viwandani Van de Vijver et al. ${ }^{13}$ conducted a survey on hypertension and found $12 \%$ prevalence with only $19 \%$ awareness on the health condition. These results are similar to those found by Ezzati et al. when looking at lifestyles and diets of people who moved from rural to urban areas in LMICs. ${ }^{14}$

There is a nutrition transition being experienced in LMICs characterised by a move from rural to urban areas and the adoption of lifestyles and diets that expose them to an increased risk of NCDs. ${ }^{15,16}$ Although supermarkets play a key role in driving the nutrition transition through increasing the availability of processed food, these same supermarkets also sell healthy food cheaply and can facilitate access to a wider range of foods. ${ }^{17,18}$ According to Rischke ${ }^{17}$ and Demmler ${ }^{18}$, Kenya has a prospering supermarket sector accounting for approximately $10 \%$ of the share of grocery sales at the national level, and it is higher in urban centres. Evidence from Kenya suggests that living near, or shopping at, a supermarket increased the consumption of processed food at the expense of unprocessed food ${ }^{17,18}$ and was associated with a higher body mass index and metabolic syndrome in adults $18,19,20$ but a lower probability of being underweight in children according to Kimenju. ${ }^{19}$ This suggests that, with appropriate interventions and support for populations, supermarkets have the potential to improve diet and health by supporting more healthful food choices. However, with the proliferation of supermarkets and nutritional transition well underway in Kenya, it is clear that a combined effort by multiple sectors is needed to achieve a change of direction that makes supermarkets a force for health.

In Kenya, it has become apparent that various societal factors, most of which do not fall within the reach of the Ministry of Health $(\mathrm{MoH})$, determine the increasing incidence of NCDs. Therefore, it is important to engage diverse sectors including agriculture, education, transport and faith especially given their impact on food production and retail, transport behaviour and the emotional/spiritual health of a population. It can be seen, therefore, that a Multi-Sectoral Approach (MSA) may be a prevention-and-control strategy that successfully engages with the complex societal problem that is NCDs. Arora et al. ${ }^{21}$ describe an MSA as other sectors of the economy working with or without the collaboration of the health sector to achieve 
health outcomes. The World Health Organisation (WHO) in the 2011 Moscow declaration notes, in addition to strengthening health systems, the importance of strengthening the role of the government and developing multi-sectoral public policies and legal frameworks for NCD prevention. As detailed by Juma et al., ${ }^{22}$ Kenya is among other LMICs such as Cameroon, Nigeria and Malawi that have adopted an MSA so as not to leave NCD prevention and control to only the Ministries of Health, which are often challenged by inadequacies of requisite infrastructure and resources.

This analysis is drawn from the baseline assessment of a natural experimental study conducted in Kisumu (an area surrounding a proposed new mall [Lake Basin Mall] containing a new supermarket) and in Homabay (an area with several existing supermarkets) both in western Kenya. In Kisumu, the formal opening of the Lake Basin Mall has been delayed following allegations - reported by the Kenyan Ethics and Anti-Corruption Commission (EACC) - that its developers inflated the construction cost by 2.5 billion Kenyan shillings (about 23.3 million USD). ${ }^{23}$ Regulatory and local community stakeholders were interviewed at each site. These stakeholders' perspectives can be understood using Bronfenbrenner's socio-ecological model, ${ }^{24}$ which was later formalised into the socio-ecological theory, widely used in deciphering human action. This model and its extrapolated theory provide a framework for understanding how multiple actors work in concert to account for an individual's behaviour. The main tenet of the model is that an individual, with their inherent biological and personal history factors, operates within a space that includes other people (family and other relationships); community settings (schools, workplaces and neighbourhoods), and societal structures (social and cultural norms) and is consequently influenced in their behaviours by their surroundings. Consequently, choosing what to eat is thus a decision to a large extent out of an individual's control, and rather a function of his or her environment. Socio-ecological models are useful in analysing health behaviours as they categorise factors that influence individual behaviours on multiple levels: intrapersonal, interpersonal, organisational, environmental and policy and legislative. Since the models focus on the importance of context in behaviour, they also highlight a variety of opportunities useful for intervening in individual behaviour.

The aim of this analysis is to identify the perceptions of interviewed stakeholders on the expected impact of the new Lake Basin Mall in Kisumu and supermarkets in Homabay on diets in the local community.

Particularly, the analysis seeks answers to the following questions:

1. What type of stakeholders are influential in the development of malls and associated food retail outlets and how were they involved?

2. How do these stakeholders view the mall development as driving (or not) economic development and its overall impact on the diets and health of the local population?

3. What predictions do they have that a new supermarket might have on local diets and other food retail activities in the area?

4. Is there evidence of multi-sectoral approach in stakeholder perspectives? 


\section{Methods}

\section{Study design and study areas}

This analysis forms part of a wider natural experimental study that was reviewed and approved by the Scientific Ethical Review Unit (SERU) of the Kenya Medical Research Institute (KEMRI, SSC 3730). As detailed in its related protocol paper, Foley et al. ${ }^{25}$ describe the study sites, Kisumu and Homabay in western Kenya, as purposely sampled because they presented suitable conditions for a natural experimental study. Kisumu study area has a soon-to-open supermarket combined with a department store (i.e. hypermarket) and other food outlets, at Lake Basin Mall located in a residential area that has not had such a mall before. Homabay County was identified as the control site as it did not have a new supermarket but had other smaller supermarkets and similar demographic characteristics to Kisumu County.

\section{Participants}

Table 1. Interview participants

\begin{tabular}{|lll|}
\hline Role & Kisumu & Homabay \\
\hline $\begin{array}{l}\text { Regulatory (legally mandated to give various certifications before business } \\
\text { premises - including food retail }- \text { can open) }\end{array}$ & 15 & 10 \\
\hline $\begin{array}{l}\text { Local Community (have no legal mandate but are influential and their input is } \\
\text { required before business premises can open) }\end{array}$ & 5 & 6 \\
\hline Total & 20 & 16 \\
\hline
\end{tabular}

The stakeholders referenced on Table 1 above were interviewed from a similar schedule developed by the researchers. This variety of selected stakeholders were drawn from the regulatory and community sectors of Kenya because they were considered important contributors to the development of the Lake Basin Mall in Kisumu and other potential retail developments in Homabay. Their positions in society enabled them to draw their mandate from the country's laws, culture or perceived widespread knowledge in the area. For instance, before starting developments such as Lake Basin Mall, the developer is required by law to seek certification from a range of legal entities representing the environment, trade and health. These constitute the 'regulatory stakeholders' of this study. The second group of stakeholders that such a developer needs approval from fall under what we term 'local community stakeholders'. These include groups of people or institutions that hold sway in the community, such as faith, business, higher education institutions and local school districts. Local community stakeholders may not have a legal mandate but are nonetheless important because they endorse and encourage local acceptability of business developments and practices. Local community stakeholders are recruited to give their views through community stakeholder forums designed to assess the impact of new developments on local residents. Reports from such forums comprise the list of documents used to get a final certification to 
operate new business premise. To avoid potential identification of stakeholders, findings were reported according to these two groupings.

Before the interviews for this study were conducted, stakeholder engagement meetings were undertaken in both study sites in November 2018. During these initial meetings, study staff explained the study objectives and sought input from the stakeholders - including how the study might be improved and who was best placed to work as data clerks in the community. It was important to draw data clerks from the community as they would be familiar with local community gatekeepers who would introduce them to participants. During this initial meeting, representatives of various sectors also identified other important community decision-makers who could contribute to the study.

\section{Data collection}

Participants in this study were stakeholders purposively selected from the initial engagement meetings and subsequently identified through a snowballing process. Participants provided written informed consent before the interviews were conducted. Stakeholders at the Kisumu site were interviewed first, followed by those in Homabay. Qualitative interviews were semi-structured (with slight variation in emphasis for the two sites) using a topic guide (Appendix 1). Questions were open-ended to allow for a discussion moderated by the study staff but led by the stakeholder.

The questions for the interviews in Kisumu sought information about the development of the Lake Basin Mall and supermarket and what they thought were the possible impacts on the diets of the local community in Kisumu. Stakeholders gave their views on the possible impacts of the mall and supermarket on local diets, the economy, way of life and other effects across the community.

The interview questions for Homabay focused on what the stakeholders thought influenced the choice of food among different age and socio-economic groups in the county and how this had changed over time with the introduction of small supermarkets. The Homabay stakeholders were also asked to make future projections on changes in local diets. Other questions dwelt on the impact of supermarkets on the local environment and economy. From the data collected at the two study sites, the main focus for this analysis was the interviewees' perceptions of the impact of the mall in Kisumu and supermarket in Homabay on local diets.

All interviews were audio-recorded and transcribed verbatim. The interviews were also translated into English as necessary since the interviews were conducted in Kiswahili and Dholuo (the national and predominant local language respectively).

\section{Data analysis}

Data derived from interviews were analysed using the NVivo software. The data collected from Kisumu was analysed using NVivo version 11 and version 12 for the same collected at Homabay. The two versions of NVivo are similar, so no special arrangement was made to combine and harmonise the themes identified. Through thematic discourse analysis, we identified themes and linked them to the data 
in an inductive manner. We sited this within a background framework of the socio-ecological model, conceptualising diet choice as being influenced by environmental determinants. We followed a stepped process that begun with reading and re-reading the transcribed interviews and coming up with codes. We combined these codes into themes that represented the data, then examined in detail which themes to report. We choose themes worthy of reporting as per our objectives and research questions.

\section{Results}

Regulatory and local community stakeholders contributed their perceptions on the expected impact of the new Kisumu mall and supermarket on local diets, and on how supermarkets in Homabay have continued to shape local diets. The two groups of stakeholders seem to be similarly concerned that supermarkets make unhealthy food items easily available for incorporation into local diets with the result that more people develop diet-related NCDs. Despite this, both groups showed their appreciation of the usefulness of supermarkets as a reliable source of food, albeit requiring that the shopper discern healthy from unhealthy food items for the purposes of keeping healthy diets. Overall, they were unsure whether the pricing in the supermarkets would be attractive enough to make the local population abandon their usual small-scale food vendors. Below are some sentiments expressed by the stakeholders across the main themes.

\section{The fear of fast food and its health implications}

With regard to how the new supermarket will impact local diets and other food retail outlets, the interviewed stakeholders had various views. Both regulatory and local community stakeholders were concerned about the health of the local population, believing that supermarkets make it easier for people to access unhealthy fast food. They were in agreement that the local diet might change when the population is exposed to fast food sold in supermarkets.

"One thing I know in a hyper supermarket, there are going to be these fast foods, and fast food are not healthy ... like French fries, sausages, fried chicken, yeah those are the ones that... those are the ones actually that sell, yeah." Regulatory stakeholder: Kisumu.

Further, both groups of stakeholders acknowledged some consequences of access to these foods to include both weight gain in childhood and the development of cardiovascular risk factors in adulthood.

"Of course the sale of these foods often leads to overweight and obesity in children. If it is in adults it could be related to maybe hypertension if you are overweight, so that can be a negative [outcome of a new supermarket." Regulatory stakeholder: Kisumu.

"...Another negative impact that we can also get because of you know... health wise you know most people use this local maybe... uh... vegetables but you know people will have access to junk foods and then there will be increase of obesity and even cardiovascular diseases." Local community stakeholder: Kisumu. 
In contrast to regulatory stakeholders, local community stakeholders noted that they would help the community avoid embracing fast-food diets through education and persuasion. In particular, faith leaders, herein included among local community stakeholders, acknowledged that they have the leeway to persuade community members to behave in a certain way. They confirmed that they would continue to do that even with the advent of readily accessible unhealthy food. Their way of thinking is evidence of how much they are in touch with their community and know what problems they are likely to encounter and some possible ways of solving these problems. This connection to their community makes them know what intervention action may or may not work for community problems.

"Yeah, we should educate our community about the importance of practicing [eating] fresh vegetable." Local community stakeholder: Kisumu.

"... It is about education of our local community, like you know in [faith community] we normally encourage our members to practice vegetable diet, but you know we normally tell them that they have freedom to choose. "Local community stakeholder: Homabay.

There was also the concern that, in addition to unhealthy foods, supermarkets may expose their customers to other unhealthy environmental factors such as air pollution. This comment likely emanates from their familiarity with toxins and contaminants that may cause disease and which the increased vehicle movement around busy shopping centres is likely to bring.

"In terms of pollution of course if you are exposed to contaminants then you might get short term or longterm diseases..." Regulatory stakeholder: Homabay.

"The negative there will be generation of waste, there will be pollution that is both noise, air and water. There might be crime, of course, any place that has development may experience [incidences of] prostitution or even theft...those are the negative that I can think of now." Regulatory Stakeholder, Kisumu.

\section{Supermarkets as reliable, but selective, food outlets}

Interviewed stakeholders had mixed reactions to the usefulness of supermarkets in the community. Some saw supermarkets as offering a reliable and dependable shopping experience as far as prices, availability and quality of food were concerned. They were in a fixed location unlike the food hawkers commonly found in these areas who were often on the move. On the other hand, other stakeholders viewed supermarkets as being expensive and thus only for the well-to-do in the community. Yet some stakeholders thought that different people would buy different things from the supermarket or their usual food retailers depending on how much money they had or just because they had chosen to for no apparent reason.

"You know in the supermarket even the prices of these commodities they are a little bit affordable, you can find that in this small kiosk a loaf of bread is fifty shillings but in the supermarket you will get it at forty-seven, so I rather walk in supermarket, go buy my loaf of bread which is forty-seven and save three shillings." Local community stakeholder: Homabay 
"They will be there still because not, not everybody living in those areas will want to go to the mall, like if you have maybe a hundred shillings and you want to go into the supermarket or that mall to buy maybe... tomatoes and you are not sure whether a kilo in that supermarket today as you are going will be a hundred bob, then you opt to buy from outside. But there are people who... don't mind about the cost of what they are going to buy in the supermarket even if it is vegetables or the herbs and spices or fruit. His or hers is to get the fruit he is looking for. So that's why it depends on the categories of people that are there." Regulatory stakeholder: Kisumu.

On the other hand, others viewed the food retail outlets as only accessible to people of 'means' and not the majority residents of the area.

"It is easier to get everything at a go then you move on [to other things] so with that advantage, you will expect a little bit of higher prices [at a supermarket]."Regulatory stakeholder: Kisumu.

Discussing how the presence of the new mall and supermarket would affect the local food traders, most regulatory stakeholders did not imagine any changes and maintained that the business returns of the local food traders would basically remain the same.

"I don't think there'll be any influence because when I look at the local I'm assuming the... the hyper market has its clientele then the local community also have their clientele that go to the food outlets, and the kind of food outlets we have in the community is different from what we are going to have in the mall..." Regulatory stakeholder: Kisumu.

\section{Perceived mixed impacts on the local community}

Interviewed stakeholders related mixed feelings about the impact of the new supermarket on local businesses and local diets. They related that the presence of the mall and supermarket in the local community was both beneficial and injurious to the local population and food retail businesses in the community.

"... You will find that now people no longer need to travel far to buy, to get some things because supermarket brought them near, secondly consumption of processed goods... you'll find a lot of processed goods in the supermarket as opposed to organic foods." Regulatory stakeholder: Kisumu.

One local community stakeholder reflected on the impacts of new supermarkets at the family level. $\mathrm{He}$ thought that the new supermarket would cause a loss of jobs when local food retailers lost their customers to the supermarkets, which he thought people would prefer. The supermarkets also do not employ as many people as the small retailers in combination do. Loss of jobs normally has a direct impact on family diet, making the family unable to purchase healthier food.

"There will be loss of jobs... because many people prefer according to me going to the supermarket than to, to open air market." Local community stakeholder: Kisumu. 
"...It may not employ as many people as small traders and at the same time it may kill those businesses of smaller traders." Regulatory stakeholder: Homabay.

However, a regulatory stakeholder thought that the new mall and supermarket would generate jobs for the local community such as in transport, banking and retail.

"... Then for some, some would benefit... if you look at the example of the [transport business] people, they are the major people who ferry people to the supermarket and away from the supermarket so for them I think the supermarket has helped them in terms of increasing their business opportunities... Then maybe things like the banks also because the supermarket is making money and I think every day they have to go and bank, so I am assuming the banks around there are also doing good business because of the returns from the supermarket, and then the security firm also they have been contracted they are also benefiting from the supermarket." Regulatory stakeholder: Kisumu.

"Maybe a few locals who are employed in the supermarket... their families are also benefiting from the income that they get from the supermarket." Regulatory stakeholder: Homabay.

On the local family level of food production, regulatory stakeholders even went further to point out that the presence of a new supermarket could threaten healthy kitchen-garden ventures of local households and replace yield from such gardens with cheap unhealthy foods.

"... They move into the supermarket and then buy readymade, so the element of buying ready-made food might probably kill our agriculture, kill our kitchen gardens. That is a negative one." Regulatory stakeholder: Homabay.

\section{Discussion}

The interviewed stakeholders perceived the impact of the Lake Basin Mall in Kisumu and supermarkets in Homabay on local diets in different ways. The prominent themes identified in our analysis of their perceptions included a fear of fast food and its health implications, for which both the regulatory and local community stakeholders were fairly in agreement. It also came out clearly that the stakeholders viewed supermarkets as reliable food outlets as they brought needed food items to local communities, though perhaps only serving a selected clientele. There was also a level of uncertainty and differences of opinion about the potential mixed impact of the mall and supermarkets on local communities and the local economy.

There was, however, a general agreement that the new supermarket at Lake Basin Mall would deepen dietary inequalities, though in a different direction than typically seen in high-income settings. It was felt that wealthier local residents would consume unhealthy fast food purchased from the new supermarket and poorer community members would continue eating from their local food vendors who sold less processed food at fairer prices. This differs from high-income countries in which healthy foods are typically viewed as more expensive than unhealthy foods. ${ }^{17,19}$ 
There is evidence of substantial multi-sectoral responsibility or at least interest in issues related to diet and health. Non-health sector stakeholders raise a range of concerns that have either direct or indirect impacts on health or the social determinants of health. Interestingly, stakeholders in the field of health did not articulate the detrimental effects of increased access to fast food as clearly as would be expected. Additionally, in reflecting on access to fast food, they demonstrated a concern for issues beyond the impacts on health such as local transport and business opportunities.

\section{Comparison with other related literature}

Even closer to the Kenyan context, Nsemenang, ${ }^{26}$ using an Afrocentric lens to interpret the socioecological model of human behaviour in the field of child development, stresses that a people use their culture (cultural constructions) to make sense of their environment. Culture is an important force that infuses its agenda on people's natural behaviours. What may not seem important to an 'outsider' could well be the point at which an intervention such as promoting healthy dietary practices may be successful. For instance, this study has shown that local community stakeholders are very important forces that shape thinking among community members. Involving them in developing an intervention targeting community health would improve its chances of success.

Multi-sectoral stakeholders interviewed in this study represent perspectives from the different socioecological levels of the community depending on the sector they were from. Their perceptions of the impact of the new mall and supermarket on diets in the local areas emanate from the layer of influence they occupy in the socio-ecological system of the community and the knowledge they have been exposed to through engaging in their environment. Their insight offers a variety of options regarding intervention points for projects that would seek to improve their community's nutrition behaviour.

Adopting a socio-ecological framework to understanding stakeholder perceptions allowed us to collect more nuanced information. From their systematic review, McCormack et al., ${ }^{27}$ in discussing health literacy in the United States of America (USA), note that the socio-ecological model allows for the interventions to improve patient literacy at various levels of action in the community as presented by the model. Additionally, Hull et al., ${ }^{28}$ in their narrative systematic review aimed at collating, summarising and synthesising evidence related to physical activity interventions aimed at young females (14-25 years) in the United Kingdom (UK), explore measures used to evaluate the intervention's impacts and recommend good practice to future similar research as best conducted using an ecological model. The preceding scholars concluded that there are multiple-level factors that act in concert in the environment and which could promote sustainable physical activity behaviour. This is similar to information presented by the regulatory and local community stakeholders who had different perspectives about the impact of supermarkets on local diets according to what they know, which in turn is influenced by their environments.

\section{Implications for policy and practice}


A multi-sectoral approach to combating NCDs works by harnessing skills and abilities found in various sectors of the country's economy and focusing this resultant synergy to fight NCDs. ${ }^{22}$ In our study, stakeholders across multiple different sectors had suggestions about how to engage in NCD prevention in the community, particularly around the availability of unhealthy food. This is the spirit on which the multi-sectoral approach is developed and thrives for the betterment of the community.

Local community stakeholders were concerned about the community's physical and mental health. In particular, faith leaders and organisations are seen as highly persuasive to the estimated $70 \%$ of Kenyans who self-describe as being religious ${ }^{29}$. They are invaluable in passing messages of healthy nutrition to the faithful and letting the food outlets know what the faithful wish they could stock. As noted by Nsamenang, ${ }^{25}$ individuals act within a cultural backdrop, so even if the cultural stakeholders do not take an active overt part in licensing business premises for operation, they tacitly decide the success of such ventures. Indeed, it is a requirement in Kenya that before a project of the magnitude of the Lake Basin Mall is developed, that officials must invite the public to give their input in a public participation workshop. At this time institutions of higher learning, faith, environment and other community stakeholders get a chance to contribute their views aimed at improving the proposed project especially on its impact on the environment and local communities. Such forums are very important and need to be emphasized and views of participants thus collected used to improve development projects. The corrupt practices often infiltrating such development projects as the Hypermarket often impede collection and use of these important views from stakeholders. The Lake Basin Mall is no exception and has had its share of corruption scandals that have seen its top developers charged in court and awaiting case verdicts according to a 2019 report by EACC. ${ }^{23}$

From the interviews, it became clear that the non-health stakeholders desired more information about the risk factors associated with NCDs. Such information would enable them to better appreciate what form their contribution to the effort to combat NCDs could potentially take, bearing in mind their differing roles and mandates. It is useful to conduct workshops to give stakeholders information about the importance of research evidence for policy decision-making.

\section{Research implications of the study}

Further work should include the assessment of the true impact of the Lake Basin Mall on the health of the surrounding population and local business opportunities as is being completed in our natural experimental study. Evaluation of the apparent early multi-sectoral stakeholder interest in health would also be of value alongside wider work exploring the stakeholder perspectives on topics of public health importance, particularly in relation to NCDs.

\section{Strengths and limitations of the study}

This is the first exploration of multi-sectoral stakeholder perspectives on a new mall and supermarket in Western Kenya. As far as we know, this is the first of this type of study; a natural experiment surveying the impact of a new mall and supermarket on the diets of local populations. This study is an important entry 
point for such studies focusing on the causes of Kenyans' changing diet behaviours. The study was limited to the western part of Kenya and further to Kisumu and Homabay towns in this expansive region. The findings only reflect hypothesised opinions of stakeholders rather than being representative of dietary changes that large food retail outlets such as malls and supermarkets have on catchment communities.

\section{Conclusion}

We explored stakeholder perspectives on the impacts of new and existing supermarkets on local diets. The prominent themes identified in our analysis were fear of fast food and health implications, supermarkets as reliable (but selective) food outlets and uncertainty and differences of opinion about the potential mixed impacts of supermarkets on local communities. We found evidence of a multi-sectoral approach with a widely held interest in potential impacts on health.

\section{Declarations}

\section{Ethical approval and consent to participate}

The study protocol was reviewed and approved by the Scientific Ethical Review Unit (SERU) of the Kenya Medical Research Institute (KEMRI SSC3730).

\section{Consent for publication}

Consent for publication was given by the KEMRI Centre for Global Health Research Scientific Committee REF: CSC NO. KEMRI/CGHR/04/05/2021. Attached.

\section{Availability of supporting data}

Data is stored securely in Kenya Medical Research Institute and University of Cambridge repositories

\section{Competing interests}

None declared

\section{Funding}

All authors are funded by The National Institute of Health Research, UK. Grant number NIHR 16/137/34 using UK aid from the UK Government to support global health research. The views expressed in this publication are those of the authors and not necessarily those of NIHR or the UK Department of Health and Social Care.

\section{Authors Contribution}


Pamela Wadende (corresponding author) led the conceptualization, initial drafting and the overall drafting process of the manuscript.

Louse Foley (senior author) supervised the conceptualisation and overall drafting process for the manuscript.

Vincent Were, Ebele Mogo, Oliver Francis, Charles Obonyo and Rosemary Musuva contributed to editing and finalisation of the manuscript.

\section{Acknowledgement}

We acknowledge the important contributions made by the participating community through their community health volunteers, field workers, study staff and the respondents. We are also indebted to Global Diet and Activity Research Group and Network (GDAR) that includes investigators and research administration staff for the support they have accorded us throughout this study.

\section{References}

1. Beaglehole R, Ebrahim E, Reddy S, et al. Prevention of chronic diseases: a call to action. Lancet 2007; 370: $2152-57$

2. World Health Organization. Global Action Plan for the prevention and control of non-communicable diseases 2013-2020. Geneva

3. Weldearegawi B, Ashebir Y, Gebeye E, et al. Emerging chronic non-communicable diseases in rural communities of Northern Ethiopia: evidence using population-based verbal autopsy method in Kilite Awlaelo surveillance site. Health Policy Planning 2013; 28: 891-898

4. Phillips-Howard PA, Laserson KF, Amek Ny, et al. Deaths ascribed to non-communicable diseases among rural Kenyan adults are proportionately increasing: evidence from a health and demographic surveillance system, 2003-2010. PLOS One 2014; 9 (11): e114010.

5. Oti SO, van de Vijver S, Kyobutungi C. Trends in non-communicable disease mortality among adult residents in Nairobi's slums, 2003-2011: applying InterVA-4 to verbal autopsy data. Glob Health Action 2014; 7: 25533

6. Dalal S, Beunza JJ, Volmink J, et al. Non-communicable diseases in sub-Saharan Africa: what we know now. Int J Epidemiol 2011; 40: 885-901

7. Etyang $A O$ and Scott JAG. Medical causes of admissions to hospital among adults in Africa: a systematic review. Glob Health Action 2013; 6: 19090

8. M'Buyamba-Kabangu J-R, Biswika RT, Thijs L, et al. In-hospital mortality among black patients admitted for hypertension-related disorders in Mbuji Mayi, Congo. Am J Hypertension 2009; 22: 64348

9. Republic of Kenya (2014). Kenya Demographic Health Survey, 2014. https://dhsprogram.com/pubs/pdf/FR308/FR308.pdf 
10. Mathenge $\mathrm{W}$, Foster $\mathrm{A}$, Kuper $\mathrm{H}$, et al. Urbanization, ethnicity and cardiovascular disease risk in a population in transition in Nakuru, Kenya: a population-based survey. BMC Public Health 2010; 10: 569

11. Joshi MD, Ayah R, Njau EK, et al. Prevalence of hypertension and associated cardiovascular risk factors in an urban slum in Nairobi, Kenya: a population-based survey. BMC Public Health 2014: 14: 1177

12. Olack $B$, Wabwire-Mangen $F$, Smeeth $L$, et al. Risk factors of hypertension among adults aged 35-64 years living in an urban slum Nairobi, Kenya. BMC Public Health 2015; 151251

13. Van de Vijver SJ, Oti SO, Agyemang C, et al. Prevalence, awareness, treatment and control of hypertension among slum dwellers in Nairobi, Kenya. J Hypertension 2013; 31: 1018-24

14. Ezzati M, Hoorn SV, Lawes CMM, et al. Rethinking the "diseases of affluence" paradigm: global patterns of nutritional risks in relation to economic development. PLoS Med 2005; 2 (5): e133

15. Steyn K, Kazenellenbogen JM, Lombard CJ, Bourne LT. Urbanization and the risk for chronic diseases of lifestyle in the black population of the Cape Peninsula, South Africa. J Cardiovasc Risk 1997; 4: $135-42$

16. Battersby J, Watson V (2018). Addressing food security in African cities. Nature Sustainability 1:153-155.

17. Rischke R, Kimenju SC, Klasen S, Qaim M. Supermarkets and food consumption patterns: The case of small towns in Kenya. Food Policy 2015; 52:9-21.

18. Demmler KM, Ecker O, Qaim M. Supermarket Shopping and Nutritional Outcomes: A Panel Data Analysis for Urban Kenya. World Development 2018; 102:292-303

19. Kimenju SC, Rischke R, Klasen S, Qaim M. Do supermarkets contribute to the obesity pandemic in developing countries? Public Health Nutr. 2015 Dec;18(17):3224-33. doi: 10.1017/S1368980015000919. Epub 2015 Apr 15

20. Demmler KM, Klasen S, Nzuma JM, Qaim M. Supermarket purchase contributes to nutrition-related non-communicable diseases in urban Kenya. PLoS One. 2017; 12(9): e0185148.

21. Arora M, Chauhan K, John S, Mukhopadhyay A. Multi-sectoral action for addressing social determinants of noncommunicable diseases and mainstreaming health promotion in national health programmes in India. Indian J Community Med. 2011;36(Suppl1):S43

22. Juma et al.(2018). Multi-sectoral action in non-communicable disease prevention policy development in five African countries BMC Public Health 2018, 18(Suppl 1):953 https://doi.org/10.1186/s12889-018-5826-6

23. EACC Press Statement, (2019). Investigation regarding allegations that the construction of the Lake Basin Development Authority (LBDA) shopping mall in Kisumu was inflated by 2.5 billion Ksh. https://eacc.go.ke/default/document/press-statement-regarding-allegations-of-inflation-by-kshs-2-5billion-in-the-construction-of-the-lake-basin-development-authority-shopping-mall-in-kisumu/

24. Bronfenbrenner. U. (1977b) Toward an experimental ecology of human development. Am Psychol. 1977;32:513-531. doi:10.1037/0003-066X.32.7.513. 
25. Foley L, Francis O, Musuva R, Turner-Moss E, Wadende P. Were V. Obonyo O. (2020). A natural experiment evaluation of the impacts of a new supermarket on dietary behaviour and the local foodscape in Kisumu, Kenya: the hypermarket, foodscape \& health study. Journal of Medical Internet Research. Preprints. 14/01/2020:17814 DOI: 10.2196/preprints. 17814

26. Nsamenang, A. B. (2015). Indigenous social science at the intersection with human development: Implications for and lessons from African Eco cultures. In L. A. Jensen (Ed.), The Oxford handbook of human development and culture: An interdisciplinary perspective (pp. 61-78). New York, NY: Oxford University Press

27. McCormack, L., Thomas, V., Lewis, M. A., and Rudd, R. (2017). Improving low health literacy and patient engagement: a social ecological approach. Pat. Edu Counsel. 100, 8-13. doi: 10.1016/j.pec.2016.07.007

28. Hull, R., de Oliveira, R., \& Zaidell, L. (2018). An Eco-logical Approach to Exploring Physical Activity Interventions Aimed at Young UK-Based Females: A Narrative Systematic Review. Psychology, 9, 2795-2823.

29. National Endowment for the Humanities https//www.africa.upenn.edu. $>\mathrm{NEH}>$ kreligion

\section{Supplementary Files}

This is a list of supplementary files associated with this preprint. Click to download.

- APPENDIX1.docx

- Wadendeetal1KEMRI.doc 